\title{
Impacto Alimentar Esofágico, \\ um Dilema Diagnóstico
}

\section{Esophageal Food Impaction, a Diagnostic Dilemma}

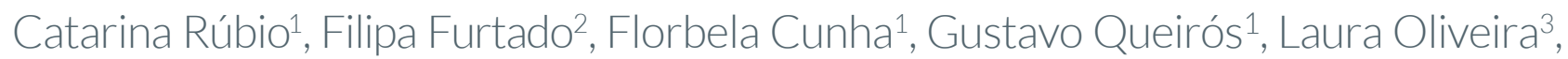
José Cabral ${ }^{3}$

\section{RESUMO}

O impacto alimentar esofágico é uma situação que na maioria dos casos é secundária a patologia esofágica congénita ou adquirida, podendo constituir a primeira manifestação da doença.

Descreve-se um rapaz, 11 anos, saudável, com sialorreia e dor retrosternal que surgiram subitamente durante o jantar. Foi submetido a endoscopia digestiva alta, que revelou um fragmento de carne impactado no terço distal do esófago (que foi extraído) e estrias longitudinais na mucosa. A análise histológica das biópsias esofágicas foi compatível com eosinofilia esofágica. Iniciou terapêutica com inibidor da bomba de protões com melhoria clínica e histológica.

O diagnóstico diferencial das principais causas de impacto alimentar associadas a eosinofilia esofágica, nomeadamente esofagite eosinofílica, doença do refluxo gastroesofágico e eosinofilia esofágica respondedora a inibidor da bomba de protões, constitui um desafio clínico.

PALAVRAS-CHAVE: Criança; Esofagite Eosinofílica/diagnóstico; Inibidores da Bomba de Protões; Perturbações da Deglutição 


\section{ABSTRACT}

Esophageal food bolus impaction is frequently secondary to congenital or acquired esophageal pathology and may constitute the first sign of the disease.

We describe a healthy 11-year-old boy, presented with sialorrhea and retrosternal pain developed suddenly while having dinner. An upper gastrointestinal endoscopy detected an impacted piece of meat in the distal third of the esophagus and longitudinal striae in the mucosa. The biopsy revealed esophageal eosinophilia. He was medicated with a proton pump inhibitor, with clinical and histological improvement.

The diagnostic differentiation between the main causes of food bolus impaction associated with esophageal eosinophilia, namely eosinophilic esophagitis, gastroesophageal reflux disease and proton pump inhibitor-responsive esophageal eosinophilia, may represent a clinical challenge.

KEYWORDS: Child; Deglutition Disorders; Eosinophilic Esophagitis/diagnosis; Proton Pump Inhibitors

\section{INTRODUÇÃO}

impacto esofágico alimentar em adultos tem uma prevalência estimada de 13 por $100000 .^{1}$ Em idade pediátrica, apesar dos dados serem mais escassos, é mais frequente na segunda infância e adolescência.

Na maioria dos casos, o impacto esofágico é secundário a patologia esofágica, como a esofagite eosinofílica (EE), doença do refluxo gastroesofágico (DRGE), estenose esofágica pós-cirurgia esofágica e dismotilidade, podendo constituir a primeira manifestação da doença. ${ }^{1,2}$

Atualmente a principal causa de impacto alimentar em crianças e adolescentes é a EE, que é uma doença crónica, imunomediada, na qual existe disfunção esofágica com infiltração eosinofílica da mucosa., ${ }^{1,3} \bigcirc$ diagnóstico de EE não é fácil, sobretudo em pediatria, e depende da presença de sintomas de dismotilidade esofágica associados a inflamação eosinofílica (> 15 eosinófilos por campo de grande ampliação (CGA) numa biópsia da mucosa) e exclusão de outras causas de eosinofilia esofágica (DRGE, eosinofilia esofágica respondedora a inibidor da bomba de protões (IBP), esofagite infeciosa, acalásia, gastroenterite eosinofílica, síndrome hipereosinofílica). Os sintomas de disfunção esofágica variam com a idade, sendo que em lactentes e crianças mais novas podem ser inespecíficos, tais como a má progressão ponderal, vómitos, dor abdominal. Nos adolescentes são mais frequentes a disfagia e o impacto alimentar. ${ }^{3-6}$

Os autores descrevem o caso de um adolescente previamente saudável que recorreu ao serviço de urgência por episódio de impacto alimentar esofágico.

\section{CASO CLÍNICO}

Trata-se de um rapaz, de 11 anos, previamente saudável e sem antecedentes familiares relevantes. Recorreu ao serviço de urgência trinta minutos após o início de quadro de sialorreia e dor retrosternal moderada, que surgiram durante o jantar quando estava a comer carne. Sem episódios anteriores de disfagia, vómitos, engasgamento, pirose, dor retrosternal ou história de atopia.

À admissão apresentava sialorreia persistente, sem sinais de dificuldade respiratória, SpO2 100\%, auscultação pulmonar com murmúrio vesicular mantido e simétrico bilateralmente. Boca e orofaringe sem alterações. Sem outras alterações valorizáveis ao exame objetivo.

Realizou radiografia torácica e cervical que não revelaram alterações. Fez diazepam 5 mg oral e ingeriu líquidos fracionados que regurgitou sistematicamente. Por ausência de resposta contactou-se Gastroenterologia Pediátrica e foi realizada endoscopia digestiva alta (EDA) que revelou estrias longitudinais e oclusão completa do esófago distal por um fragmento de carne (Fig. 1). Dois terços foram extraídos por ansa diatérmica e o restante foi empurrado para a cavidade gástrica. Na EDA não se observou, a nível esofágico, edema, anéis, exsudado, erosões ou úlceras. Efetuaram-se biópsias do terço superior, médio e distal do esófago. $\bigcirc$ estudo histológico mostrou um marcado infiltrado por eosinófilos (> 15 eosinófilos/CGA) no terço distal do esófago.

Foi medicado com esomeprazol 20 mg duas vezes por dia e foi encaminhado para consulta. Após três meses de terapêutica manteve-se assintomático e fez controlo endoscópico com biópsias esofágicas, gástricas e duodenais. A endoscopia revelou uma mucosa esofágica normal e o exame histológico não revelou alterações.

No seguimento em consulta realizou ainda avaliação analítica com hemograma, que não revelou eosinofilia, e estudo alargado de alergias alimentares com valores normais (Tabela 1).

Dois meses após a suspensão da terapêutica com IBP começou a referir episódios intermitentes de disfagia. Foi submetido novamente a EDA que revelou estrias 
TABELA 1. Parâmetros analíticos avaliados.

\begin{tabular}{|l|c|}
\hline $\begin{array}{l}\text { Parâmetros analíticos } \\
\text { (valores de referência) }\end{array}$ & Valores \\
\hline Leucócitos (5-13 $\times 10^{9}$ uL) & 5,8 \\
\hline Eosinófilos (0-6\%) & 3 \\
\hline Imunoglobulina E (IgE) (0-200 KUI/L) & 2,1 \\
\hline IgE leite* & $<0,01$ \\
\hline IgE bacalhau* & $<0,01$ \\
\hline IgE trigo* & $<0,01$ \\
\hline IgE clara de ovo* & $<0,01$ \\
\hline IgE gema de ovo* & $<0,01$ \\
\hline Ige amendoim* & $<0,01$ \\
\hline IgE soja* & $<0,01$ \\
\hline IgE avelã* & $<0,01$ \\
\hline IgE amêndoa* & $<0,01$ \\
\hline IgE noz* & $<0,01$ \\
\hline IgE atum* & $<0,01$ \\
\hline IgE camarão* & $<0,01$ \\
\hline$* 0,35$ KU/L Clsse & \\
\hline
\end{tabular}

*<0,35 KU/L Classe 0; 0,351-0,7 KU/L Classe 1; 0,71-3,5 KU/L Classe 2, 3,51-17,5 KU/L Classe 3; 17,51-50 KU/L Classe 4, 50,01$100 \mathrm{KU} / \mathrm{L}$ Classe 5; >100 KU/L Classe 6.

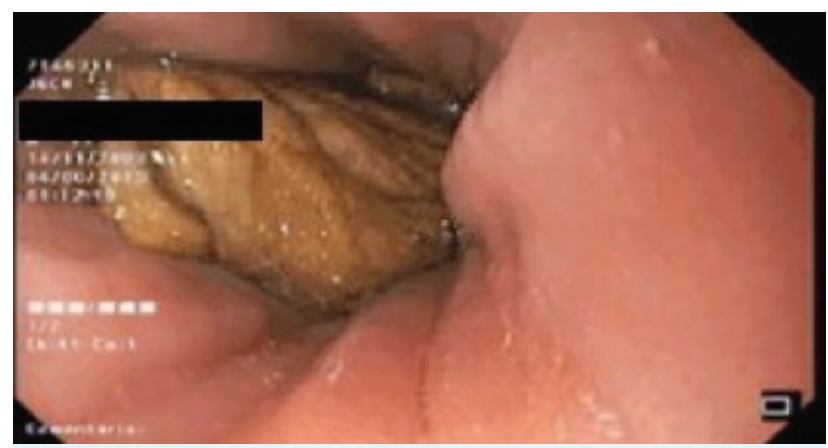

FIGURA 1. Endoscopia digestiva alta. Observa-se impacto alimentar.

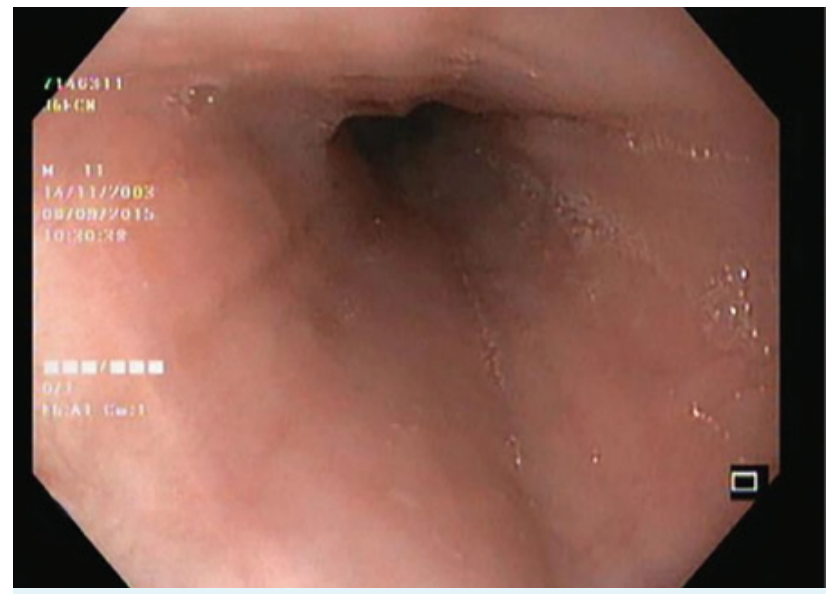

FIGURA 2. Endoscopia digestiva alta onde se observam ligeiras estrias longitudinais no terço médio do esófago. longitudinais no terço distal do esófago (Fig. 2) e mais de 15 eosinófilos/CGA no exame histológico, reiniciando IBP, que mantém.

\section{DISCUSSÃO}

Perante um quadro caracterizado por aparecimento súbito de dor torácica, disfagia e sialorreia persistente que surge no decorrer de uma refeição é obrigatório pensar em impacto alimentar. 2,5

A extração do alimento, face à presença de obstrução esofágica completa, sugerida por sialorreia e disfagia para líquidos, é emergente. Assim, deve ser realizada EDA idealmente até duas horas desde o início da sintomatologia e no máximo até seis horas depois. Em todos os casos de impacto alimentar os doentes devem ser referenciados para uma consulta de Gastroenterologia Pediátrica e realizar EDA com biópsias dos vários segmentos do esófago, gástricas e duodenais para identificação de uma possível patologia subjacente. ${ }^{1,7}$

Na presença de eosinofilia esofágica (> 15 eosinófilos/ (GA), no resultado histológico, os diagnósticos mais prováveis são a EE, a DRGE e a eosinofilia esofágica respondedora a IBP. Quando existe uma resposta clínica e histológica à terapêutica com IBP em doentes com impacto alimentar e eosinofilia esofágica, como no caso clínico apresentado, o diagnóstico de eosinofilia esofágica respondedora a IBP ou doença do refluxo gastroesofágico deve ser considerado., $3,5,8$

Habitualmente, a DRGE é acompanhada de sintomatologia de refluxo, alterações endoscópicas (úlceras, inflamação da mucosa exclusiva do terço distal) e alterações na pHmetria. ${ }^{4}$ No presente caso clínico, o doente não apresentava sintomatologia sugestiva de refluxo, nem foram observadas alterações endoscópicas compatíveis com esse diagnóstico.

Por outro lado, a eosinofilia esofágica respondedora a IBP é uma situação clínica que cursa com sintomatologia, alterações endoscópicas e histológicas sobreponíveis às da EE. No entanto, estes doentes tendem a resolver a sua eosinofilia esofágica após um período de tratamento com IBP e não apresentam patologia atópica associada, ${ }^{4}$ tal como no caso clínico descrito.

O mecanismo fisiopatológico da eosinofilia esofágica respondedora a IBP ainda não está totalmente esclarecido, existindo duas teorias que podem explicar esta entidade. A primeira tem como base a presença de DRGE, em que o epitélio esofágico é danificado pela exposição ao ácido, com consequente aumento da sua permeabilidade e possibilidade da penetração de alergénios com 
posterior recrutamento de eosinófilos nesse local. Nesta sequência, vários autores sugerem a realização de pHmetria em casos selecionados, havendo, no entanto, estudos que têm demonstrado que esta não constitui um fator preditivo para resposta a IBP. A outra teoria está relacionada com a possível ação anti-inflamatória dos IBP no epitélio esofágico e no bloqueio da secreção da eotaxina-3 que apresenta um papel determinante no desenvolvimento da EE. ${ }^{4,5,8}$

Atualmente não se sabe se a eosinofilia esofágica é uma variante da DRGE, da EE ou se é uma entidade clínica distinta, sendo a atitude terapêutica variável em cada uma das situações.,8-10 Na EE o tratamento baseia-se essencialmente em estratégias dietéticas enquanto na DRGE e na eosinofilia esofágica respondedora a IBP o tratamento de primeira linha são os IBP.

Um estudo realizado por Dohil et al, em doentes pediátricos, revelou que a resposta aos IBP em doentes com eosinofilia esofágica respondedora a IBP pode ser temporária, tal como sucedeu neste doente, pelo que são necessários mais estudos de seguimento a longo prazo para melhor caracterização desta situação clínica. ${ }^{3,8}$

O diagnóstico diferencial entre EE, eosinofilia esofágica respondedora a IBP e DRGE e a possibilidade de sobreposição destas entidades constitui um verdadeiro desafio. São necessários mais estudos para melhor esclarecimento tanto da EE como da eosinofilia esofágica respondedora a IBP, com intuito de melhorar a atitude diagnóstica e terapêutica em casos como o apresentado, que são cada vez mais frequentes.

CONFLITOS DE INTERESSE: Os autores declaram não ter qualquer conflito de interesse na realização do presente trabalho.

FONTES DE FINANCIAMENTO: Não houve qualquer fonte de financiamento na realização do presente trabalho.

CONFIDENCIALIDADE DOS DADOS: Os autores declaram ter seguido os protocolos da sua instituição acerca da publicação dos dados de doentes.

PROTEÇÃO DE PESSOAS E ANIMAIS: Os autores declaram que os procedimentos seguidos na elaboração do presente trabalho estão em conformidade com as normas das comissões de investigação clínica e de ética, bem como da declaração de Helsínquia e da Associação Médica Mundial.

CONFLICTS OF INTEREST: The authors declare that they have no conflicts of interest.

FINANCIAL SUPPORT: This work has not received any contribution, grant or scholarship.
CONFIDENTIALITY OF DATA: The authors declare that they have followed the protocols of their work center on the publication of data from patients.

PROTECTION OF HUMAN AND ANIMAL SUBJECTS: The authors declare that the procedures followed were in accordance with the regulations of the relevant clinical research ethics committee and with those of the Code of Ethics of the World Medical Association (Declaration of Helsinki).

\section{REFERÊNCIAS}

1. Kramer R, Lerner D, Lin T, Manfredi M, Shah M, Stephet T, et al. Management of ingested foreign bodies in children: a clinical report of the NASPGHAN Endoscopy Committee. J Pediatr Gastroenterol Nutr. 2015;60:562-74.

2. Sahn B, Mamula P, Ford C. Review of foreign body ingestion and esophageal food impaction management in adolescents. J Adolesc Health. 2014;55:260-6.

3. Papdopoulou A, Koletzko S, Heuschkel R, Dias JA, Allen KJ, Murch S, et al. Management Guidelines of Eosinophilic Esophagitis in Childhood. J Pediatr Gastroenterol Nutr. 2014;58:107-18.

4. Dellon ES, Gonsalves N, Hirano I, Furuta GT, Liacouras CA, et al. ACG Clinical Guideline: Evidenced Based Approach to the Diagnosis and Management of Esophageal Eosinophilia and Eosinophilic Esophagitis (EoE). Am J Gastroenterol. 2013;108:679-92.

5. Munday W, Zhang X. Proton pump inhibitor responsive esophageal eosinofilia, a distinct disease entity? World J Gastroenterol. 2014;20:10419-24.

6. Molina-Infante J, Katzka DA. Proton-pump inhibitor-responsive esophageal eosinofilia. Curr Opin Gastroenterol. 2014.30:428-33.

7. Tringali A, Thomson M, Dumonceuau J, Tavares M, Tabbers M, Furlano R, et al. Pediatric gastrointestinal endoscopy: European Society of Gastroeintestinal Endoscopy/ESGE) and European Society for Paediatric Gastroenterology Hepatology and Nutrition (ESPGHAN) Guideline Executive summary. Endoscopy. 2017;49:83-91.

8. WolfWA, Dellon ES. Eosinophilic esophagitis and proton pump inhibitors: controversies and implications for clinical practice. Gastroenterol Hepatol. 2014;10:427-32.

9. Dohil R, Newbury RO, Aceves S. Transient PPI responsive esophageal eosinophilia may be a clinical sub-phenotype of pediatric eosinophilic esophagitis. Dig Dis Sci. 2015;60:3181-93.

10. Eluri S, Dellon ES. PPI-responsive esophageal eosinophilia and eosinophilic esophagitis: More similarities than difference. Curr Opin Gastroenterol. 2015; 31:309-15. 